\title{
BER analysis of amplify-and-forward relaying FSO systems using APD receiver over strong atmospheric turbulence channels
}

\author{
Huu Ai Duong ${ }^{1}$, Van Loi Nguyen ${ }^{2}$ \\ ${ }^{1}$ Department of Electronics and Telecommunications, Korea Vietnam Friendship Information \\ Technology College, Vietnam \\ ${ }^{2}$ Department of Information Technology, Korea Vietnam Friendship Information Technology College, Vietnam
}

\section{Article Info}

Article history:

Received Jul 21, 2018

Revised Apr 11, 2019

Accepted Apr 21, 2019

\section{Keywords:}

Amplify-and-Forward

APD

Atmospheric turbulence

FSO

QAM

\begin{abstract}
In this paper, we theoretically analyze the performance of amplify-andforward (AF) serial relaying free-space optical (FSO) systems using avalanche photodiodes (APD) and subcarrier quadrature amplitude modulation (SC-QAM) over strong atmospheric turbulence channels modelled by gamma-gamma distribution. More specifically, an average bit error rate (BER) of system is theoretically derived talking into account APD shot noise, thermal noise as well as the impact of the number of AF relaying stations and turbulence. The numerical results show that using AF relay stations can extend the transmission distance and help to improve performance of FSO system significantly when compared with the direct transmission. Moreover, the selection of APD gain value is indispensable to the system performance. The proposed system could be achieved the best performance by selecting an optimal APD gain value. In addition, the optimal value of APD gain also significantly depends on various conditions, such as the number of relay stations, APD receiver noise and link distance.
\end{abstract}

Copyright (c) 2019Institute of Advanced Engineering and Science. All rights reserved.

\section{Corresponding Author:}

Duong Huu Ai,

Department of Electronics and Telecommunications,

Korea Vietnam Friendship Information Technology College,

450 Tran Dai Nghia St., Danang, Vietnam.

Email: huuai11@gmail.com

\section{INTRODUCTION}

Free-space optics (FSO) is known as green communication technology, in recent years for a lot of applications because of their ability to unlimited bandwidth, licensing-free requirements, high security, costeffectiveness and simplicity of communication system design and deployment [1-2]. Although FSO communication system are many advantages like that, but the performance of FSO systems strongly depends onatmospheric turbulence conditions, which is cause by the temperature and pressure inhomogeneity of the atmosphere [3], when an optical beam traversing the atmosphere. It will negatively effects of scattering, absorption and turbulence result in power loss and the link distance limited over several kilometers [4].

In addition, to improve the low link distance transmission over the turbulence channel, relaying FSO systems have been proposed to extend the longer transmission and mitigate the turbulence included fading [5]. Outage performance of multihop FSO systems over strong turbulence channels modelled by the gamma-gamma distribution, has been studied in [6-7]. Most recently performance of relaying FSO systems over atmospheric turbulence channels has been studied focus on Amply-and-Forward (AF) and Decode-andForward (DF) relaying FSO systems. The aim of this study, we theoretically analyze the performance FSO scheme AF serial system. In the AF relaying method, the receiver signal from source at each node will be 
amplified before retransmitted to next relay node and repeated through intermediate nodes to the destination node, and AF relaying method is a potential solution to increase link distance transmission.

Previous works, FSO systems have mainly been implemented by employing OOK or PPM modulation techniques for commerce because of the simplicity and low cost for OOK and superior power efficiency for PPM modulation. However OOK modulation needs an adaptive threshold that is difficult adjustment to accomplishin the presence of atmospheric turbulence, also PPM modulation technical has a poor bandwidth efficient and require high transceiver synchronization [8-9]. To overcome the limitations of OOK and PPM, subcarrierintensity modulation quadrature amplitude modulation (SC-QAM) has been recently proposed. The performances of FSO system using SC-QAM over lognormal and gamma-gamma turbulence channels have been found in some recent studies [10-12]. Most recently, ASER performance analysis of MIMO/FSO systems using SC-QAM signaling over atmospheric turbulence channelshave been reported [13].

Others technique can significantly improve the performance of FSO systemin receiver path is use avalanche photodiodes (APDs). APD are widely used because they provide higher values ofresponsivity compared with PIN photodiodes. The characteristics of APD devices and the performance of APD-based receivers have beenstudied extensively [14-16]. The use of APD for the cases of FSO systems using QAM modulations was reported in [17]. Furthermore APD-based Amplify-and-Forward Serial Relaying FSO Systems has been studied in [18]. There has been, however, no study on BER analysis of AF relaying FSO system using APD receiver.

In this paper, we therefore analyze BER of AF relaying FSO/SC-QAM system using APD receiver over strong atmospheric turbulence channel. Moreover we obtain important performance for the practical system design, such as the effects of APD gain, transmitted power and link distance on the BER of FSO/SC-QAM systems over strong atmospheric turbulence channel.

\section{SYSTEM DESCRIPTIONS}

\subsection{AF relaying FSO system using QAM signal}

We start by investigating a typical serial an APD-based AF relaying FSO system, in which signal from the source node is transmitted to the destination through $N$ relaying nodes serially as shown in Figure 1. The source node, relaying node, and destination node schemes are illustrated in Figure 2.In Figure 2a, which system was employed SC-QAM, electrical data from the signal source is firstly up-converted to an intermediate frequency $f_{c}$, this electrical subcarrier QAM signal is then used to modulate the intensity of a Laser.

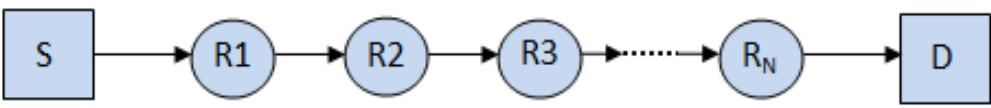

Figure1. An example of a serial relaying FSO communication system

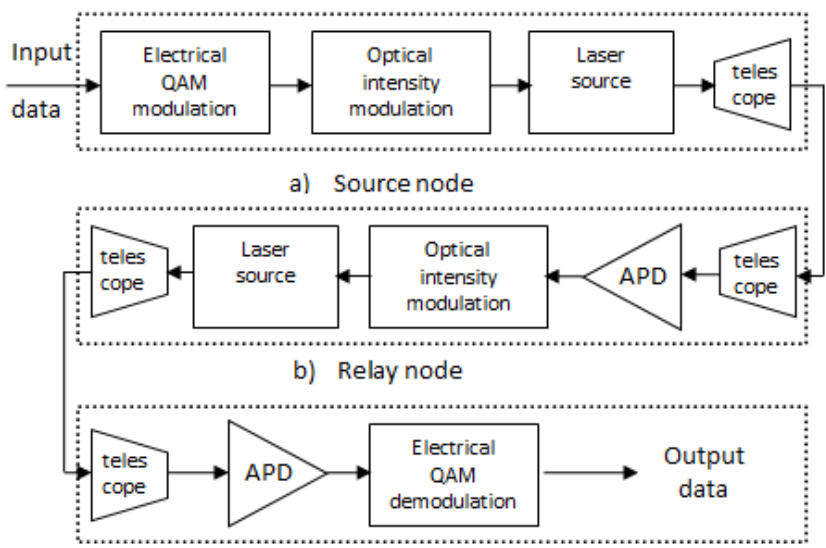

c) Destination node

Figure 2. The source node, relaying node and destination node of FSO/QAM systems 
The electrical SC-QAM signal at the output of QAM modulator can be written as:

$$
e(t)=s_{I}(t) \cos \left(2 p f_{c} t\right)-s_{Q}(t) \sin \left(2 p f_{c} t\right)
$$

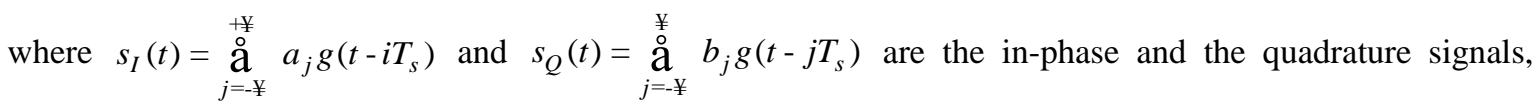
respectively, $a_{j}(t), b_{j}(t)$ are respectively the in-phase and the quadrature component information amplitudes of the $j-t h$ transmitted data symbol, $g(t)$ is the shaping pulse and $T_{S}$ denotes the symbol interval.

The QAM signal is then used to modulate the intensity of an electrical-to-optical (E/O) laser before pointing laser beam through a telescope of the transmitter to the relaying node, the transmitted signal can be expressed as:

$$
s(t)=P_{s}\left\{1+k\left[s_{I}(t) \cos \left(2 p f_{c} t\right)-s_{Q}(t) \sin \left(2 p f_{c} t\right)\right]\right\}
$$

where $P_{S}$ denotes the average transmitted optical power per symbol at each hop and $k$ is the modulation index, $0<k<1$. Due to the effects of link loss $a_{1}$ and $X_{1}(t)$ - the random process for the signal scintillation caused by atmospheric turbulence of the first hop, the received optical signal at the first relay node can be expressed as

$$
r_{1}(t)=a_{1} X_{1}(\mathrm{t}) P_{s}\left\{1+k\left[s_{I}(t) \cos \left(2 p f_{c} t\right)-s_{Q}(t) \sin \left(2 p f_{c} t\right)\right]\right\}
$$

At each relay node, an APD receiver is used for amplifying signal as shown in Figure $2 b$. The received optical signal is firstly converted into electrical signal and then amplified by the APD, after has been amplified by the APD, this signal is used to re-modulate intensity of laser source and relayed to the next relay node. Due to slow turbulence changes, the DC component $\left\{a_{1} X_{1}(t) P_{S}\right\}$ can be filtered out by a bandpassfilter. Therefore the electrical signal output of AF module at the first relay node will be

$$
e_{1}(t)=\bar{g} \hat{\mathrm{A}} \mathrm{a}_{1} X_{1}(\mathrm{t}) P_{s} k e(t)+n_{1}(t)
$$

where $\bar{g}, \hat{\mathrm{A}}, n_{1}(t)$ are theaverageAPD gain, responsivity of photodiode and receiver noise at the first node, respectively.The total receiver noise $n_{1}(t)$ at the first node consisting of APD shot noise, thermal noise and can be modelled as a stationary random Gaussian process whose variance is given by[19]

$$
s_{1}^{2}=2 q \bar{g}^{2} \hat{\mathrm{A}} F_{A} P_{S} k \mathrm{D}_{f} a_{1} X_{1}+\frac{4 k_{B} T}{R_{L}} F_{n} \mathrm{D}_{f}
$$

where $k_{B}, T, R_{L}, F_{n}, q, \Delta f, F_{A}$ represent the Boltzmann constant, the absolute temperature of receiver, the APD's load resistance, the amplifier noise figure, the electron charge the symbol's effective noise bandwidth and the exceed noise factor. Where $\Delta f=R_{b} / 2 \log _{2}(M)$ with $R_{b}$ is the bit rate of the system and $M=M_{I} \times M_{Q}$ and $F_{A}=k_{A} \bar{g}+\left(1-k_{A}\right)(2-1 / \bar{g})$ with $k_{A}$ denoting the ionization factor.

With AF relaying scheme, relay node first normalizes the received optical signal in (4) to unity and then is optically modulated with power $P_{S}$ in order to transmitted power per symbol at the $i-t h$ node equal with transmitted power per symbol at the source and re-transmitted to the next node. The transmitted optical signal at the first node therefore can be expressed as (11) [18]

$$
s_{1}(t)=P_{s}\left\{1+X_{1}(t) k e(t)+\frac{n_{1}(t)}{\hat{\mathrm{A}} \bar{g} a_{1} P_{S}}\right\}
$$

Repeating such steps above through the number of relay stations, $N$, the electrical signal in the output of APD at the destination node can be derived as following 


$$
e(t)=\bar{g} \hat{\mathrm{A}} P_{s} a_{N+1} \tilde{\mathrm{O}}_{i=1}^{N+1} X_{i}(t) k e(t)+\stackrel{\mathrm{a}}{i=1} \frac{n_{i}(t) a_{N+1} \tilde{\mathrm{O}}_{j=i+1}^{N+1} X_{j}(t)}{a_{i}}
$$

where $a_{i}, X_{i}(t)$ are the path loss and atmospheric turbulence of the connection between the $(i-1)-t h$ and $i-t h$ node respectively. Whereas $n_{i}(t)$ is the receiver noise at the $i-t h$ node. Also the variance of total accumulated receiver noise at the destination node is given by

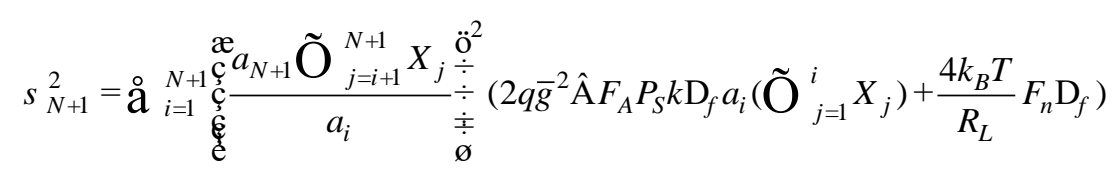

Similar to (7) and (8), the instantaneous electrical SNR per symbol, $\gamma$, is the random variable at the output of the APD, as a function of instantaneous fading value $X$, with system and channel parameters can be expressed as follows

$$
g=\frac{\left(\bar{g} \hat{\mathrm{A}} P_{s} k a_{N+1} \tilde{\mathrm{O}}_{i=1}^{N+1} X_{i}(t)\right)^{2}}{s_{N+1}^{2}}=\frac{\left(\bar{g} \hat{\mathrm{A}} P_{s} k a_{N+1} X^{N+1}\right)^{2}}{s_{N+1}^{2}}
$$

\subsection{Channel model}

When an optical wave beam go through the free space atmosphere, it experiences the deformation caused various atmospheric condition, such as scattering, refraction, and absorption between the terminals of FSO link. In this study, we consider the factors characterizing an FSO channel including link loss, and atmospheric turbulence. The link loss is caused by both molecular absorption and aerosol scattering in the air. The total link loss of system can be expressed as

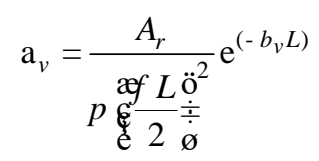

where $A_{r}, \phi, L, B_{v}$ denote the area of the receiver aperture, the optical beam's divergence angle in radian, the link lengthbetween the source and destination and the atmospheric extinction coefficient, respectively [20].

The atmospheric turbulence caused the irradiance fluctuation represents signal scintillation. There are many distribution models to describe the atmosphere-induced turbulence. For strong turbulence condition, it is generally accepted that $X(t)$ is a random process with gamma-gamma distribution. The probability density function (pdf) of the irradiance intensity in the strong turbulent is given by [13].

$$
f_{X}(X)=\frac{2(\alpha \beta)^{\frac{\alpha+\beta}{2}}}{\Gamma(\alpha) \Gamma(\beta)} X^{\frac{\alpha+\beta}{2}-1} K_{\alpha-\beta}(2 \sqrt{\alpha \beta X})
$$

where $\Gamma($.$) is the Gamma function and K_{\alpha-\beta}($.$) denotes the modified Bessel function of the second$ kind of order $(\alpha-\beta)$. The positive parameter $\alpha$ represents the effective number of large-scale cells of the scattering process, and the positive parameter $\beta$ represents the effective number of small-scale cells of the scattering process in the atmospheric. It can be shown that the parameters $\alpha$ and $\beta$ are directly related to atmospheric conditions through the following expressionswhere $\sigma_{S}$ is the scintillation index, and is defined at [13] as $\sigma_{S}=\exp \left(\omega_{1}+\omega_{2}\right)+1$; where

$$
\alpha=\left[\exp \left(\frac{0.49 \sigma_{2}^{2}}{\left(1+0.18 d^{2}+0.56 \sigma_{2}^{12 / 5}\right)^{7 / 6}}\right)-1\right]^{-1}
$$




$$
\beta=\left[\exp \left(\frac{0.51 \sigma_{2}^{2}\left(1+0.69 \sigma_{2}^{12 / 5}\right)^{-5 / 6}}{1+0.9 d^{2}+0.62 \sigma_{2}^{12 / 5}}\right)-1\right]^{-1}
$$

In these equations, $d=\sqrt{k D^{2} / 4 L}$ where $k=2 \pi / \lambda$ is the optical wave number, $\lambda$ is the wavelength, $L$ is the link distance, and $D$ is the radius of a circular receiving aperture, and $\sigma_{2}$ is the Rytov variance, and assuming spherical wave propagation, defined as:

$$
s_{2}^{2}=0.492 C_{n}^{2} k^{7 / 6} L^{11 / 6}
$$

In (14), $C_{n}^{2}$ is the refractive-index structure parameter, which is weather depended.

At the AF system, the probability distribution function of atmospheric turbulence thought $N$ nodes $\left(X_{a}\right)$ for FSO system is given in (15) [21].

$f_{X}(X)=\frac{2(\alpha \beta)^{\frac{\alpha+\beta}{2}}}{(N+1) \Gamma(\alpha) \Gamma(\beta)} X^{\frac{\alpha+\beta}{2}-N-1} K_{\alpha-\beta}(2 \sqrt{\alpha \beta X})$,

\section{BER ANALYSIS}

In this section, we will analyze the BER performance of the AF relaying FSO/QAM system using APD receiver over strong atmospheric turbulence channel. The mathematical formula to perform bit error rate of QAM modulation can be found at:

$$
B E R=\grave{\mathrm{O}}_{0}^{¥} B E R_{\text {inst }} f_{X}(x) d x
$$

In which $B E R_{\text {inst }}$ instantaneous bit error rate of system in destination node, for the $M_{I} \times M_{Q}$ rectangular QAM modulation, the instantaneous BER is given as

$$
B E R_{\text {inst }}=\frac{1}{\log _{2}\left(M_{I} M_{Q}\right)}\left(\stackrel{\circ}{\log _{2=1}\left(M_{I}\right)} P_{M_{I}}(k)+\stackrel{\circ}{\log _{2}\left(M_{Q}\right)} P_{M_{Q}}(1)\right)
$$

where $P_{M_{I}}(k)$ and $P_{M_{Q}}(l)$ are respectively denoted the BER occurring on the $k-t h$ bit of $M_{I}$-ary PAM and the $l-t h$ bit of $M_{Q}$-ary PAM. Using the derivation of the probability that the $k-t h$ bit in error for the $I$-ary PAM, $P_{M_{i}}$ and $P_{M_{q}}$ can be respectively given by

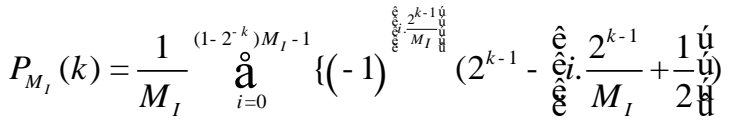

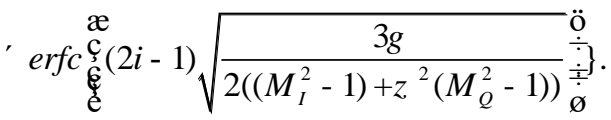

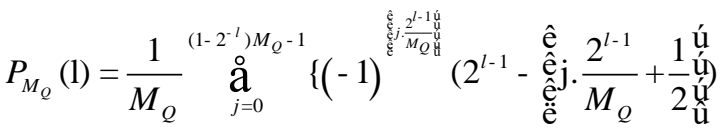

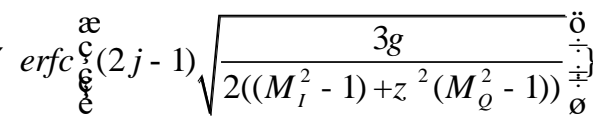


In the (18), (19) $\gamma$ and $\zeta$ are the instantaneous electrical SNR per symbol and the quadrature to inphase decision distance ratio with $\zeta=d_{Q} / d_{I}$. Replace (18), (19) with the parameter SNR $\gamma$ calculated in (9) into (17), we have the instantaneous BER for rectangular QAM/FSO system for AF relaying using APD receiver. And then BER can calculate by (16) with $f_{X}(X)$ for the gamma-gamma channel given in (15). Define $P_{I}$ and $P_{Q}$ as

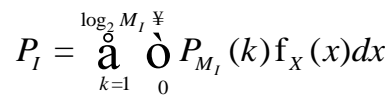

$$
\begin{aligned}
& P_{Q}=\stackrel{\mathrm{o}}{\mathrm{o}}_{l=1}^{\log _{Q} M_{Q}} \underset{0}{\mathrm{O}} P_{M_{Q}}(1) \mathrm{f}_{X}(x) d x
\end{aligned}
$$

The BER of FSO system can be written as

$$
B \mathrm{ER}=\frac{1}{\log _{2}\left(M_{I} M_{Q}\right)}\left(P_{I}+P_{Q}\right)
$$

\section{NUMERICAL RESULTS}

In this section, using previous derived expression, we present numerical results for BER analysis of AF relaying FSO/QAM system using APD receiver over strong atmospheric turbulence modeled by the gamma-gamma distribution. In our analysis, for fair comparison, BER is calculated as the function of the transmittedpower per bit $P_{b}=P_{t} / \log _{2}(\mathrm{M})$ with $P_{t}$ is total transmitted power per symbol at source and $N$ nodes. Also the denotes the average transmitted optical power per symbol at each node $P_{s}=P_{t} /(N+1)$ so the relationship between $P_{b}$ and $P_{s}$ can be written as $P_{s}=P_{b} \log _{2}(M) /(N+1)$. The performance analysis is carried out under the influence of different operating condition, which is include the average APD's gain and thermal, the number of relay node, link distance. Relevant parameter consider in our analysis are provided in Table 1.

Table 1. System parameters and constants

\begin{tabular}{lll}
\hline Parameters & Symbol & Value \\
\hline Operational wavelength & $\lambda$ & $1550 \mathrm{~nm}$ \\
Photodiode responsivity & $\hat{\mathrm{A}}$ & 1 \\
APD's resistance & $R_{L}$ & $1000 \Omega$ \\
Ionization factor & $\mathrm{k}_{\mathrm{A}}$ & 0.7 \\
Amplifier noise figure & $F_{N}$ & 2 \\
Boltzmann constant & $k_{B}$ & $1.38 \times 10^{-23}$ \\
Modulation index & $\kappa$ & 1 \\
Electron charge & $\mathrm{q}$ & $1.6 \times 10^{-23} \mathrm{C}$ \\
Index of refraction structure & $C_{n}^{2}$ & $9.10^{-14} \mathrm{~m}^{-2 / 3}$ \\
Link distance & $L$ & $1 \div 8 \mathrm{~km}$ \\
Aperture diameter & $D$ & $0.08 \mathrm{~m}$ \\
Atmospheric extinction coefficient & $\beta v$ & $0.1 \mathrm{~dB} / \mathrm{km}$ \\
Beam's divergence angle & $\phi$ & $5 \mathrm{mrad}$ \\
Decision distance ratio & $\zeta$ & 1 \\
\hline
\end{tabular}

Figure 3 show the system BER versus average APD gain $\bar{g}$ with direct transmission and different relay stations, the number relay stations $N=1,2,3$ and 4 are used in analysis, the transmitted power per bit $P_{b}=12 \mathrm{dBm}$ link distance $L=6000 \mathrm{~m}$, bit rate $R_{b}=2 \mathrm{Gbps}, T=300 \mathrm{k}$ and using $8 \times 4 \mathrm{QAM}$ scheme. As the figure clearly show, BER parameter improves significantly with increasing the number of relay station, and better than direct transmission. For more importantly, it is also seen that the selection of APD's gain $\bar{g}$ has a significant impact on the system performance. More clearly, to reach the lowest BER value, the optimal gain value $\bar{g}$ from 6 to 12 with different relay stations. Also the BER value will decrease when the average APD gain pass over the optimal gain value.

Figure 4 illustrates the BER performance of systems as a function of the average APD gain, for different values of link distance $L=4000 \mathrm{~m}, 6000 \mathrm{~m}, 8000 \mathrm{~m}$. The number relay stations $N=3, P_{b}=8 \mathrm{dBm}$, $T=300 \mathrm{~K}, R_{b}=2 \mathrm{Gbps}$ are used in this analysis. As you can see, increasing transmission link distance impact to system performance. More clearly, an increase in $L$ from $4000 \mathrm{~m}$ to $6000 \mathrm{~m}$ and $8000 \mathrm{~m}$ results in a 
significant bit error performance degradation. For example, the best BER value reached approximately $10^{-9}$ at $L=4000 \mathrm{~m}$ with optimal gain $\bar{g}=6$ and reached from $8 \times 10^{3}$ to $10^{-2}$ at $6000 \mathrm{~m}$ and $8000 \mathrm{~m}$ with $\bar{g}=6$ and $\bar{g}=10$ respectively. The optimal gain varies between 6 and 10 .

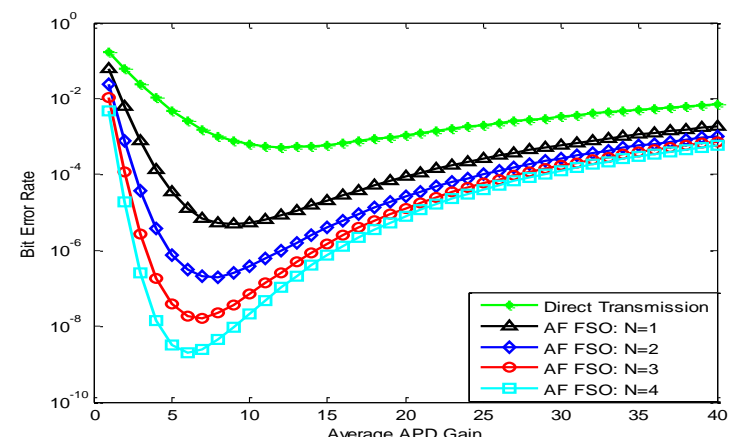

Figure 3. BER versus average APD gain $\bar{g}$ with different relay stations, transmitted power per bit $P_{b}=12 \mathrm{dBm}, L=6000 \mathrm{~m}, R_{b}=2 \mathrm{Gbps}, T=300 \mathrm{~K}$, and $8 \times 4$ QAM scheme with $N=1,2,3$ and 4

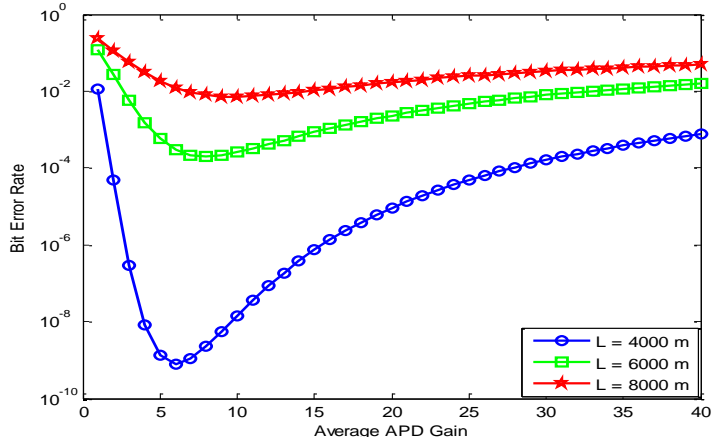

Figure 4. BER versus Average APD for values of $L$ using $8 \times 4$ QAM, $N=3, P_{b}=8 \mathrm{dBm}, T=300 \mathrm{~K}$,

$$
R_{b}=2 \mathrm{Gbps}
$$

In Figure 5, we further analysis the effect of temperatures noise on the system performance with transmitted power per bit $P_{b}=3 \mathrm{dBm}, R_{b}=3 \mathrm{Gbps}, L=2000 \mathrm{~m}, N=1$. As the figure clearly illustrates, when the degree of receiver increases lead to increase the thermal noise, therefore BER of system will be increased too. BER value reach to $10^{-11}$ at $T=300 \mathrm{~K}$ and decrease to $2 \times 10^{-8}$ at $T=1100 \mathrm{~K}$. In addition, it is shown the impact of temperatures noise on the selection of optimal gain. It is seen that the optimal gain changes more significantly when the receiver temperature varies. The optimal gain also varies from 6 to 10 when the receiver temperature increases between $300 \mathrm{~K}$ and $1100 \mathrm{~K}$.

Figure 6 shown the system BER versus transmitted power per bit $P_{b}$ at the link distance $L=6000 \mathrm{~m}$ and optimal gain $\bar{g}=6$ is selected. The figure illustrates, when the directly transmission from source to destination node, the transmitted power per bit require to reach $B E R=10^{-8}$ about $19 \mathrm{dBm}$. With the $N$ relay stations, we can obtain performance improvements of $4 \mathrm{~dB}, 6 \mathrm{~dB}, 7 \mathrm{~dB}$ for $N=1,2$ and 3 in comparison with direct transmission. Furthermore, we can see that, with the same transmitted power per bit requirement, while increasing the relay station $(N=4,5)$, the system performance did not increasing significantly. So in this case, at the link distance $L=6000 \mathrm{~m}$ we should use maximum $N=3$ relay nodes to reach the best BER value and reduce system and deployment cost.

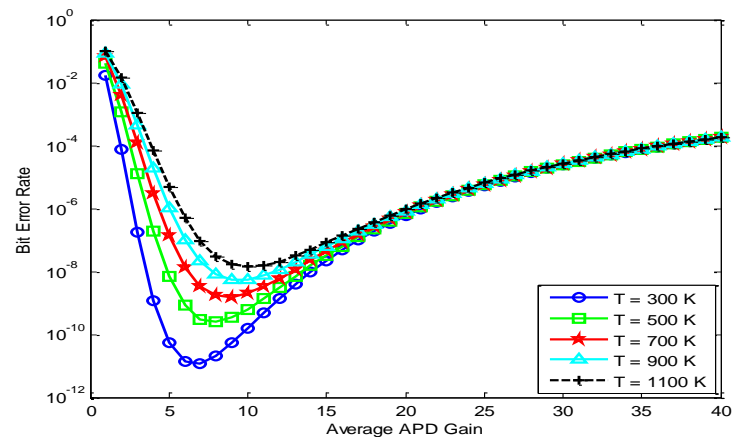

Figure 5. BER versus average APD gain for $8 \times 4$

QAM with different temperatures noise and transmitted power per bit $P_{b}=3 \mathrm{dBm}, R_{b}=2 \mathrm{Gbps}$ $N=1, L=2000 \mathrm{~m}$

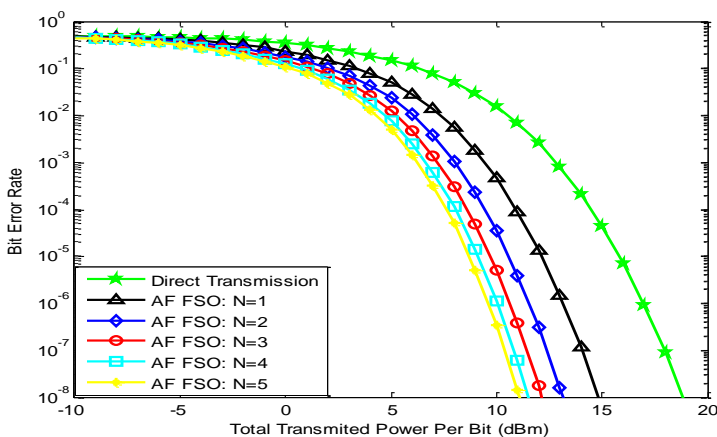

Figure 6. BER versus the transmitted power per bit $P_{b}$ with different relay stations, $N=1,2,3,4$ and 5, optimal gain $\bar{g}=6, \mathrm{~L}=6000 \mathrm{~m}, \mathrm{~T}=300 \mathrm{k}$,

$\mathrm{Rb}=2 \mathrm{Gbps}$ and $8 \times 4 \mathrm{QAM}$ scheme 


\section{CONCLUSION}

In this paper, we have analysis the bit error rate of amplify-and-forward relaying for FSO/SC-QAM scheme using APD receiver over gamma-gamma atmospheric turbulence channel. We have derived theoretical expressions for AF FSO/SC-QAM system considering with different relay stations, link distance, the average APD's gain and thermal. We clearly seen a significantly improvement when deploying relay technique in comparison with direct transmission. Furthermore, the numerical results showed that using APD with proper selection of optimal gain could improve the system performance greatly, and in this work, the optimal gain to reach the best BER of AF FSO/QAM system could vary from 6 to 10 for typical APD receiver.

\section{REFERENCES}

[1] V. W. S. Chan., "Free-space optical communications," J.Lightwave Technol., vol. 24, no. 12, pp. 4750-4762, 2006.

[2] A. Majumdar and J. Ricklin., "Free-Space Laser Communications: Principles and Advances," Optical and Fiber Communications Series, Springer Media, 2008.

[3] X.Zhu and J.M.Kahn., "Free-space Optical communication through atmospheric turbulence channels," IEEE Trans. Commun., vol.50, no.8, pp.1293-1300, Aug. 2002.

[4] Varanasi Sri Lalitha Devi(1), SubbaSrujanaSree(2), SistuRajani(3), Varanasi BharathiSeshasai., "Effects of weak atmospheric turbulence on FSO link Systems and its reducing technique," International Journal of Advancements in Research \& Technology, Volume 2, Issue 11, November-2013.

[5] M. Safari, and M. Uysal., "Relay-Assisted Free-Space Optical Communication," Wireless Communication, IEEE Trans., vol.7, no. 12, pp. 5441-5449, Dec. 2008.

[6] T. A. Tsiftsis, et al., "Multihop Free-Space Optical Relaying Communications Over Strong Turbulence Channels," IEEE Int.Conf. on Communications, vol. 6, pp. 2755-2759, 2006.

[7] C. K. Datsikas, et. al., "Serial Free-Space Optical Relaying Communications Over Gamma-Gamma Atmospheric Turbulence Channels," IEEE/OSA J. of Optical Communications and Networking, vol. 2, no. 8, pp. 576-586, Aug. 2010.

[8] W. Huang, J. Takayanagi, T. Sakanaka, M. Nakagawa., "Atmospheric optical communication system using subcarrier PSK modulation,” IEICE Trans. Commun., vol.E76-B, no.9, pp.1169-1177, Sept. 1993.

[9] S. G. Wilson, M. Brandt- Pearce, Q. Cao, and 1. H. Leveque., "Freespace optical MIMO transmission with Q-ary PPM," IEEE Trans. Commun., vol. 53, Issue 8, pp. 1402-1412, Aug. 2005.

[10] Kostas P. Peppas and Christos K. Datsikas., "Average Symbol ErrorProbability of General-Order Rectangular Quadrature Amplitude Modulation of Optical Wireless Communication Systems Over Atmospheric Turbulence Channels," J. Opt. Commun. Netw., Vol. 2,Issue 2,pp. 102- 110,Feb. 2010.

[11] Xuegui Song, MingboNiu, Julian Cheng., "Error Rate of SubcarrierIntensity Modulations for Wireless Optical communications," IEEE CommunLetters, Vol. 16,Issue 4,pp. 540-543,Apr. 2012.

[12] Md. Zoheb Hassan, Xuegui Song, and Julian Cheng., "Subcarrier Intensity Modulated Wireless Optical Communications with RectangularQAM," J. Opt. Commun. Netw., Vol. 4,Issue 6,pp. 522-532,Jun. 2012.

[13] Duong Huu Ai, Ha DuyenTrung and Do Trong Tuan., "Pointing Error Effects on Performance of Amplify-andForward Relaying MIMO/FSO Systems Using SC-QAM Signals Over Log-Normal Atmospheric Turbulence Channels," the 8th Asian Conference on Intelligent Information and Database Systems (ACIIDS), vol. 9622 of LNAI, Springer-Verlag, pp. 607-619, Mar. 2016.

[14] Conradi, J., "Distribution of gains in avalanche photodiodes-experimental results," IEEE Trans. Electron. Devices, ED-6, (6), pp. 713-718, 1972.

[15] Webb, D., "Properties of avalanche photodiodes," RCA Rev., 35, pp. 234-278, 1974.

[16] Sorensen, N., Gagliardi, R., "Performance of optical receivers with APD, " IEEE Trans. Commun., COM-27, (11), pp. 1315-1321, 1979.

[17] Bach T. Vu, Ngoc T. Dang, Truong C. Thang, Anh T. Pham., "Bit error rate analysis of rectangular QAM/FSO systems using an APD receiver over atmospheric turbulence channels,"IEEE/OSA Journal of Optical Communication and Networking, Vol.5, issue 5, page 437-446.

[18] Thanh V. Pham, et al., "APD-based Amplify-and-Forward Serial Relaying FSO Systems over Gamma-Gamma Channels," IEEE Fifth International Conference on Communications and Electronics (ICCE), October 2014.

[19] G. P. Agrawal., "Fiber-Optic Communication Systems," New York: Wiley-Interscience, 2002.

[20] S. Karp., "Optical Channels: Fibers, Clouds, Water, and the Atmosphere." Plenum, 1988.

[21] Duong Huu Ai., "Average Channel Capacity of Amplify-and-Forward MIMO/FSO Systems over Atmospheric Turbulence Channels," International Journal of Electrical and Computer Engineering (IJECE), Vol. 8, No. 6, pp. 148- 153, Dec. 2018. 


\section{BIOGRAPHIES OF AUTHORS}

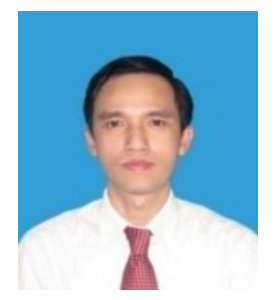

Huu Ai Duong, received BS degree in Radio and Telecommunications from Hue University of Sciences, Vietnam, in 2003, and M.E degree of Electronic engineering from Danang University of Technology, Vietnam, in 2011, and PhD of Electronic and Telecommunications in Hanoi University of Technology, Vietnam, in 2018. Currently, he is a lecturer at the Korea Vietnam friendship Information Technology College, Danang, Vietnam. His research interests include optical wireless communications, radio over fiber, and broadband networks.

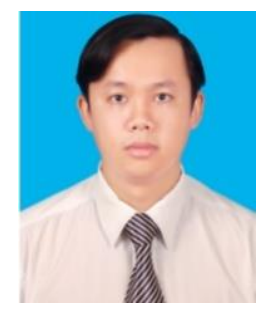

Van Loi Nguyen, he received his Master of Engineering in Computer Science from the University of Danang, Vietnam in 2010, a Ph.D. degree from Soongsil University in 2017. He is currently a lecturer at Korea - Vietnam Friendship Information Technology College. His research interests include multimedia, information retrieval,artificial intelligence, and database. 